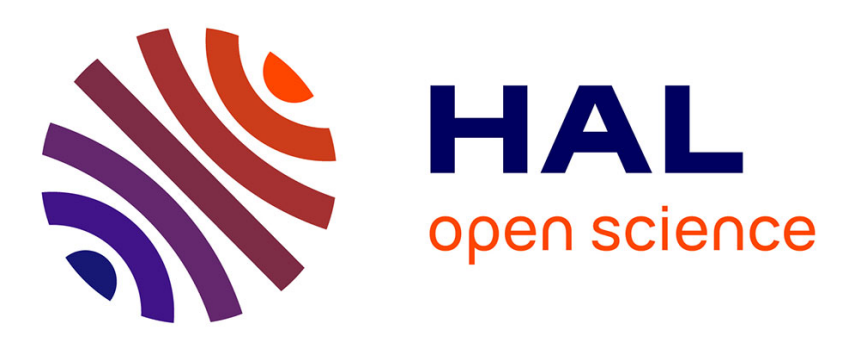

\title{
Hippocrate : critique de la faculté de soigner
} Thibaut de Saint Maurice

\section{To cite this version:}

Thibaut de Saint Maurice. Hippocrate : critique de la faculté de soigner. Multitudes, 2021, Lignes décoloniales, 84, pp.177-183. 10.3917/mult.084.0177 . hal-03531949

\section{HAL Id: hal-03531949 https://hal.science/hal-03531949}

Submitted on 18 Jan 2022

HAL is a multi-disciplinary open access archive for the deposit and dissemination of scientific research documents, whether they are published or not. The documents may come from teaching and research institutions in France or abroad, or from public or private research centers.
L'archive ouverte pluridisciplinaire HAL, est destinée au dépôt et à la diffusion de documents scientifiques de niveau recherche, publiés ou non, émanant des établissements d'enseignement et de recherche français ou étrangers, des laboratoires publics ou privés. 


\title{
Hippocrate : critique de la faculté de soigner
}

\author{
Thibaut de Saint Maurice \\ ISJPS UMR 8103, Panthéon-Sorbonne \\ ERC DEMOSERIES
}

Fallait-il être particulièrement obsessionnel ou voyeur pour se lancer dans le visionnage de la deuxième saison de la série Hippocrate ${ }^{1}$, en pleine troisième vague de l'épidémie de Covid-19 ? Depuis plus d'un an, en effet, l'hôpital public occupe le centre de la scène médiatique : la saturation de ses services de réanimation est surveillée comme le lait sur le feu, la fatigue des équipes soignantes fait l'objet de nombreux reportages tandis que les médecins deviennent les invités récurrents des émissions d'actualités. Ce à quoi l'on peut ajouter la manière dont l'hôpital s'est imposé dans l'expérience de dizaines de milliers de malades et de proches de malades. On n'a donc jamais autant parler de I'hôpital et des soignants et ils n'ont jamais été aussi présents au cœur des conversations ordinaires, jusqu'aux applaudissements, informels et éphémères, du printemps dernier. Dans ce contexte particulier, que peut-on attendre d'une série comme Hippocrate dont l'ambition affichée est de plonger le spectateur dans la réalité de l'hôpital aujourd'hui, en suivant, au plus près, le parcours de jeunes internes en stage ? Qu'est-ce que cette série peut encore nous donner à voir que nous n'ayons déjà vu ?

La question se pose d'autant plus, que, le contexte pandémique mis à part, la série Hippocrate est l'adaptation/déclinaison d'un long-métrage de cinéma éponyme, sorti en 2014. Que peut donc la série, que le cinéma ne pouvait pas ? A quoi bon remettre en œuvre, une histoire et un univers déjà raconté et qui plus est par le même auteur ? La forme sérielle permet-elle de jeter un autre regard sur une même réalité ?

Enfin, la question rebondit encore dès lors que l'on s'intéresse au genre dans lequel s'inscrit Hippocrate. Depuis plusieurs années, les séries médicales ont ouvert les portes des hôpitaux pour les spectateurs et leur ont donné à voir, non seulement l'administration des soins, mais aussi les coulisses de la vie hospitalière. Ainsi au sein du genre des séries médicales, quelle est la projection singulière de la série de Thomas Lilti ?

Si la diffusion de la deuxième saison d'Hippocrate intervient effectivement dans le contexte de la pandémie due à la Covid-19, la série a commencé bien avant, puisque la première saison a été diffusée en 2018. Et dans cette deuxième saison, pourtant tournée dans les premiers mois de la pandémie, la réalité de la situation sanitaire est tenue à distance. Jusqu'aux 10 dernières minutes du dernier épisode, il n'est pas question de la Covid-19, ni même d'un nouveau virus qui sévirait à l'étranger. La fiction présente ainsi une chronologie alternative, qui produit un fort effet de contextualisation et qui offre

\footnotetext{
${ }^{1}$ Hippocrate est une série télévisée créée par Thomas Lilti en 2018 et diffusée par Canal+
} 
donc un premier cadre d'interprétation pour le spectateur, à propos de la crise sanitaire. Le récit qu'elle déploie a commencé avant la pandémie et finit par nous y conduire, alors même qu'entre-temps, entre ces deux premières saisons, la pandémie a surgi dans le réel des spectateurs. Le récit se poursuit donc après mais comme ce qui continuait de se passer avant, ce que confirme Thomas Lilti : "Ce qu'on raconte, c'est l'état de l'hôpital juste avant la crise. $\|^{2}$ La série toute entière change de statut et s'impose comme la séquence manquante du grand récit médiatique autour de l'hôpital qui vient de se déployer dans les média d'actualité tous ces derniers mois. En racontant l'avant - presque à la manière d'un prequel - Hippocrate permet aux spectateurs de comprendre que la crise sanitaire tient aussi à la crise structurelle qui touche l'hôpital public en France. Et quand l'épidémie surgit enfin dans la fiction, c'est bien l'ensemble du récit précédent qui se trouve alors repositionné comme le contexte singulier qui permet de comprendre pourquoi cette épidémie place l'hôpital sous tension. Ainsi, bien qu'il se défende d'avoir voulu " instrumentaliser la crise en intégrant le Covid-19 dans la série " parce que " cela aurait été un peu obscène. " ${ }^{3}$ Thomas Lilti exprime néanmoins la possibilité singulière qu'à une fiction sérielle de constituer une ressource pour interpréter le réel qu'elle décrit, comme cause du réel vécu par le spectateur. Ici le réalisme de la série ne tient donc pas seulement à la vraisemblance de sa représentation, mais aussi à la manière dont elle transforme la perception de la réalité par le spectateur.

Mais ce qu'il y a de singulier avec cette série, c'est qu'elle procède d'une vision bien antérieure encore. Dès 2014, avec ce premier long-métrage de cinéma sorti sous ce même titre, Hippocrate, Thomas Lilti s'engage sur le chemin de la critique de l'institution hospitalière. Le film suit deux jeunes internes, pendant quelques semaines de leur apprentissage dans un service de médecine interne. Les décisions qu'ils prennent conduisent à deux dysfonctionnements majeurs : une faute médicale entraîne la mort d'un patient mais se retrouve couverte par les médecins référents, tandis qu'une initiative des deux jeunes internes pour soulager une patiente est lourdement punie. L'adaptation en série reprend ces situations mais en les démultipliant. La sérialité permet d'augmenter le nombre de personnages, parce que sa plus longue durée, favorise un attachement à un collectif de personnages. ${ }^{4}$ L'on passe ainsi des deux internes du film - Benjamin et Abdel - à au moins quatre dans la saison 1 de la série, et au moins cinq dans la saison 2 dont le récit va suivre le parcours. Sans compter le personnel infirmier et aide-soignant qui n'occupe toujours pas le devant de l'intrigue mais qui, dans la série, du fait même du plus grand développement de la fiction, prend une place plus importante. Ainsi l'adaptation sérielle du long-métrage permet la mise en scène d'un collectif plus fourni de personnages

\footnotetext{
2 "Thomas Lilti, réalisateur : " Je ne voulais pas instrumentaliser la crise en intégrant le Covid-19 dans Hippocrate ", propos recueillis par Audrey Fournier, Le Monde, 3 avril 2021

${ }^{3}$ Idem

${ }^{4}$ de Saint Maurice T. , " Portrait du sériephile en philosophe » in Le pouvoir des liens faibles, Gefen A. Laugier S., (sous la dir.), 2020, CNRS éditions
} 
afin d'explorer plus en détail les différentes difficultés rencontrées par ces hommes et ces femmes qui soignent les autres. Mais l'adaptation sérielle signifie aussi un changement esthétique. Entre innovation et répétition, la forme sérielle, comme le montre Umberto Eco, développe une esthétique de la variation ${ }^{5}$. Plusieurs motifs sont repris au fur et à mesure des épisodes, mais sans qu'ils le soient à l'identique. Les variations ainsi produites introduisent une répétition singulière propre à représenter la vie ordinaire ${ }^{6} \mathrm{~d}^{\prime}$ un hôpital. Les journées s'enchaînent, rythmées par des procédures régulières : le point sur les dossiers, les consultations, les prescriptions, les entrées et les sorties des malades, la préparation des gardes de nuit, comme autant de variations des procédures de la veille. Même dans le contexte extraordinaire de chaque début de saison - la quarantaine des médecins titulaires dans la saison 1 et le transfert du service des urgences en médecine interne à cause d'une inondation au début de la saison 2 - les épisodes s'enchaînant, parviennent à raconter la banalité de la vie à l'hôpital, celle qui constitue la trame de fond des situations extraordinaires qui y sont vécues : le manque de café dans la salle de repos, un dossier qui s'égare malgré le système de classement par pochettes de couleurs, les pauses cigarettes ou sucreries sur le palier de l'escalier de secours, la fatigue et le doute qui s'emparent des internes, à la fin de la journée, dans le silence de la chambre de garde. Le traitement sériel du " sujet " d'Hippocrate - le long-métrage - apporte donc un regard différent sur le parcours de ces jeunes médecins en devenir et permet de raconter que ce n'est pas l'extraordinaire des situations vécues à l'occasion de la prise en charge de tel ou tel malade qui use ces personnages, mais plutôt l'ordinaire d'une vie hospitalière qui ne laisse aucune place à la possibilité d'une attention à leur égard. Et c'est ce motif-là, celui de cette souffrance des soignants qui surgit au sein même de l'institution censée soigner la souffrance, qui se donne peu à peu à voir dans la série et qui se dessine comme le regard propre de la série Hippocrate au sein du genre auquel elle appartient.

En tant que fiction suivant l'apprentissage de jeunes médecins, dans le décor d'un ancien hôpital public, Hippocrate s'inscrit en effet dans le genre déjà largement constitué de la série médicale. On y retrouve l'attention portée aux internes, déjà présente dans la série américaine Grey's Anatomy ${ }^{7}$ et l'attention portée à la vie d'un service hospitalier, déjà présente dans Urgences ${ }^{8}$ par exemple. Hippocrate s'inspire largement de ces deux séries, on y retrouve des situations communes, des personnages communs et jusqu'à certaines mises en scènes profondément déterminées par des éléments du " décor hospitalier ":

\footnotetext{
${ }^{5}$ Eco, Umberto. «Innovation et répétition : entre esthétique moderne et post-moderne », Réseaux, vol. 68, no. 6, 1994, pp. 9-26.

${ }^{6}$ Au sens par exemple que dégage Sandra Laugier dans « Emerson : penser l'ordinaire ", Revue française d'études américaines, vol. n91, no. 1, 2002, pp. 43-60.

7 Grey's Anatomy est une série créée par Shonda Rhimes en 2005, toujours en cours de production, et diffusée aux États-Unis par la chaine ABC et en France par TF1.

${ }^{8}$ Urgences est une série créée par Michael Crichton, produite entre 1994 et 2009 et diffusée aux États-Unis sur la chaîne NBC et en France par France 2.
} 
le couloir, la chambre, la double-porte battante, le brancard...Ces références plus ou moins explicites et assumées participent résolument de l'inscription d'Hippocrate dans le genre et l'éloigne de son origine cinématographique. En tant que série médicale, Hippocrate participe donc au travail du genre dans lequel elle s'inscrit et qui consiste précisément à offrir au spectateur, la possibilité d'un inventaire critique du lieu de savoir et de pouvoir qu'est l'hôpital, au sens où Foucault en menait l'analyse archéologique dans Naissance de la clinique ${ }^{9}$. Le savoir médical est représenté mais pour être doublement questionné : non seulement dans sa pertinence thérapeutique mais aussi dans sa légitimité institutionnelle. Les jeunes internes de la série, Hugo, Alyson, Chloé et Arben découvrent ainsi que leurs connaissances médicales ne suffisent pas à prendre soin des patients, voire que la seule mobilisation de ces connaissances constitue un obstacle aux soins qu'ils tentent d'administrer. Mais ils se heurtent aussi aux limites de l'institution hospitalière, en la découvrant régie par des rapports de force entre services, des objectifs économiques incompatibles avec une délivrance convenable des soins et par des effets de structure plaçant le soignant en position de fragilité. Ainsi la voix singulière d'Hippocrate dans le genre de la série médicale est-elle à chercher dans la mise en scène de la souffrance au travail, de ces jeunes soignants en plein apprentissage, jusqu'à la mise en péril de leur propre vie. A la fin de la première saison, Chloé fait un arrêt cardiaque dans sa voiture et, comme un motif qui commence à se dessiner, à la fin de la deuxième saison, c'est Igor qui se retrouve en réanimation après qu'on le soupçonne d'avoir voulu mettre fin à ses jours. Au sein du genre de la série médicale, Hippocrate emprunte donc autant qu'elle apporte, pour donner à voir l'angle mort de l'hôpital : une institution entièrement dévolue au soin des malades et qui pourtant ne prend pas soin de ceux qui soignent, malades qu'ils sont de ne pouvoir effectivement prendre soin de ceux qui leur sont confiés.

Ainsi, regarder la deuxième saison d'une série médicale comme Hippocrate, en pleine crise sanitaire ne relève pas d'une " obsession voyeuriste " pour le spectacle médical. Le récit qu'elle déploie et les personnages qu'elle développe, permettent aux spectateurs, épisode après épisode et dans cet enchaînement si particulier entre les deux saisons, d'approcher l'ordinaire de la vie hospitalière et du « devenir médecin » de jeunes internes en plein apprentissage de leur faculté de soigner. Car tel est au fond ce que la série donne à voir que nous n'avions pas déjà vu sous cette forme : la critique de la faculté de soigner de la médecine hospitalière. Et il faut entendre ici " critique " au sens kantien du terme, comme une réflexion sur les limites de la raison à l'œuvre dans la pratique effective de la médecine aujourd'hui. Critique au sens kantien du terme encore, dans la capacité de la série à rendre sensible, les « antinomies " de la raison médicale. Certes l'analyse n'est pas aussi strictement rigoureuse que celle que mène Kant ${ }^{10}$, mais il s'agit bien dans la série

\footnotetext{
${ }^{9}$ Foucault, M. Naissance de la clinique, Paris, 2015, Puf, " Quadrige ».

${ }^{10}$ Kant, Critique de la raison pure, Paris, GF, $2^{\mathrm{ème}}$ partie, $2^{\text {ème }}$ division "Dialectique transcendantale ".
} 
de montrer des contradictions insolubles de la raison médicale telle qu'elle se déploie à I'hôpital aujourd'hui, et de faire de la tension de ces contradictions, le ressort nonseulement narratif, mais aussi esthétique d'Hippocrate, puisque ce sont ces contradictions qui sont variées et entremêlées, au fil des épisodes et au fil des deux saisons. On peut identifier au moins deux de ces " antinomies » qui concernent respectivement les jeunes internes entre apprentissage et responsabilité et le travail du soin, entre compétence technique et compétence morale.

La première de ces antinomies, c'est celle qui concerne les internes que la série place au centre de l'attention. Chloé, Hugo, Alyson et Arben ont certes des parcours différents, mais quand la série débute, ils sont bien internes tous les 4 . Et rapidement ils vont tous travailler dans le même service de médecine interne, dont les médecins titulaires ont été exclus pour avoir été en contact avec un virus inconnu. En tant qu'interne, ils sont à la fois en apprentissage, pas tout à fait médecin encore et, à la fois, en situation d'exercer des soins médicaux et de prescrire, donc déjà tout à fait médecin. La contradiction est là, bien réelle, dramatisée par le confinement des médecins titulaires : les internes ne sont pas tout à fait des médecins et les internes sont des médecins. Perdus entre les deux états, ils apprennent à devenir médecins sous la pression de l'exigence d'être déjà ce qu'ils sont en train de devenir. Cette contradiction qui surgit dès le premier épisode entraîne tout le récit. Chaque interne en présente une variation possible : Chloé est la plus expérimentée par exemple, c'est elle qui organise la vie du service dans le $1^{\text {er }}$ épisode. Elle est donc assurément médecin, parce qu'elle est assurément responsable, clairvoyante et compétente pour organiser la continuité des soins dont les patients ont besoin en l'absence des médecins référents. Mais toute professionnelle du soin qu'elle semble être, elle est incapable de diagnostiquer les risques qu'elle prend pour sa santé, jusqu'à subir les terribles conséquences d'un arrêt cardiaque, quelques épisodes plus tard. Quant à Arben, interne en médecine légale au début de la série, il est rapidement détaché en médecine interne pour renforcer l'équipe. Il est techniquement très fort, sait pratiquer des soins que les autres ne savent pas pratiquer, il est engagé auprès des patients et gagne le respect de tous. Il est là encore assurément, déjà, un médecin idéal alors qu'il n'est pas encore titulaire. Et pourtant, on apprend 7 épisodes plus tard, qu'il n'est précisément pas médecin, puisqu'il n'a pas fini ses études et qu'il exerce donc la médecine sans diplôme. Démasqué à la fin de la saison 1, il ne refait son apparition au début de la saison 2 qu'à l'occasion d'une nouvelle situation extraordinaire qui lui permet, pendant quelques épisodes d'exercer de nouveau cette médecine qu'il incarne parfaitement.

Très directement, cette antinomie de l'apprentissage et de la responsabilité dans laquelle les internes se trouvent pris au piège, révèle une vulnérabilité qu'ils sont incapables de prendre en charge seuls. En revanche, à mesure qu'ils apprennent à travailler ensemble dès lors qu'ils se rapprochent et développent une action collective et concertée ils parviennent non seulement à soulager pour chacun d'eux, la pression de la contradiction 
de leur condition, mais aussi à soigner effectivement les malades qui leur sont confiés. Se dessine ainsi, par la solidarité et le travail d'équipe, une solution à cette première antinomie. Comme dans le deuxième épisode de la saison 1 où Alyson, Chloé Hugo et Arben, tous les 4 réunis auprès d'un patient alcoolique, parviennent à stopper une hémorragie interne. Ou toujours dans le même épisode, lorsque Alyson se met en tête de retrouver le dentier d'une patiente jetée par mégarde dans les poubelles de l'hôpital et que ses co-internes la rejoignent et l'aident à mettre la main dessus. Retrouver un dentier ce n'est pas directement un geste médical, mais c'est une attention au besoin singulier d'une patiente et cela constitue assurément une autre manière d'en prendre soin.

Précisément, la deuxième antinomie que donne à voir la série est celle qui concerne la double finalité du travail de soin. Soigner c'est à la fois guérir au sens d'administrer une thérapeutique efficace pour lutter contre les effets d'une pathologie et c'est à la fois prendre soin d'un malade au sens du développement d'une attention à même de répondre à l'expression du besoin singulier d'un malade ${ }^{11}$. C'est le double travail du cure, compétence technique et du care, compétence morale, face auquel les jeunes internes de la série se trouvent démunis. A n'envisager le travail du soin que comme une technique thérapeutique, le médecin développe une certaine efficacité mais traite ses patients comme des cas et non plus comme des hommes et des femmes singulièrement vulnérables. Mais à l'inverse, l'empathie n'est pas toujours bonne conseillère et l'attention portée à la situation particulière d'un malade, par exemple, une jeune fille hospitalisée à la suite d'une tentative de suicide, peut conduire un médecin, le jeune interne Hugo, à commettre une faute grave en enfreignant le protocole de soin, ce qui donne à cette jeune fille la possibilité de réitérer sa tentative de suicide. Mais surtout la série adresse cette antinomie à un niveau supérieur en mettant en scène de soignants dont personne ne prend soin. Malgré la proximité dans le travail, malgré la convivialité des repas et des fêtes dans le réfectoire des internes, ces jeunes médecins en devenir sont seuls avec leurs souffrances. Dans la saison 2, lorsqu'lgor, interne aux urgences, comprend qu'il a oublié une patiente dans un box, cela l'affecte sans qu'il ne trouve de ressource autour de lui pour s'en remettre et finit par se suicider. A l'annonce de l'accident, le Dr Brun, chef de service, décide de fermer les urgences. Comment pourrait-il continuer de soigner des patients dès lors qu'il n'est pas capable de prendre soin des siens ? Seul le surgissement de l'épidémie de Covid-19, faisant effraction dans la fiction, peut forcer le dernier épisode de la saison à se prolonger quelques minutes encore. Aucune solution à cette antinomie-là n'est esquissée dans la série. Sa tension est au plus haut point après deux saisons et 16 épisodes. Mais après autant de temps passé à suivre ces personnages et à les voir se débattre avec ces contradictions, c'est l'attention des spectateurs qui peut - qui doit ? - prendre le relais. "Les personnages de fiction télévisée (...) peuvent être lâchés et ouverts à l'imagination et à l'usage de chacun, confiés à nous, comme s'il restait

\footnotetext{
${ }^{11}$ Molinier P., Paperman P., Laugier S., ( dir) Qu'est-ce que le care ?, Paris, 2009, Petite bibliothèque Payot
} 
à chacun d'en prendre soin..$^{12}$ ". Puisque l'hôpital ne parvient pas à prendre soin de ses soignants, c'est à la société qui l'entoure de prendre soin d'eux. La portée critique de la fiction trouve ici son horizon politique et moral. Comme une invitation à transformer notre idée de l'hôpital comme " machine à guérir ${ }^{13}$ " pour reconnaître plutôt les contradictions de ses idéaux et la vulnérabilité des praticiens qu'elles révèlent.

La vision centrale de la série Hippocrate s'exprimait au fond dès le générique: une succession de photographies plus ou moins anciennes, plaçant en son centre, la figure d'un soignant. Si donc cette série est visionnaire, ce n'est pas parce qu'elle prédit quoi que ce soit, mais c'est parce qu'elle donne à voir ce que même la crise sanitaire n'a pas permis de bien voir : la grande vulnérabilité des soignants. Tous ces derniers mois de pandémie, la fatigue voire le désarroi des soignants a bien occupé le devant de la scène médiatique, mais pour être immédiatement transformé en signe de leur dévouement, de leur professionnalisme et finalement de leur héroïsme. Or ce n'est pas tant de l'admiration de la société que l'hôpital et les soignants ont besoin, mais d'une prise en charge effective de leur vulnérabilité. Pour cela, une série comme Hippocrate, aussi importante soit-elle, ne suffit pas. Mais elle ouvre une voix et exprime à sa manière l'exigence formulée par Hippocrate : "faire le bien ou au moins, ne pas faire de mal $^{14}$ ".

Cet article a reçu un financement du Conseil européen de la recherche (CER) dans le cadre du programme de recherche et d'innovation Horizon 2020 de l'Union européenne (convention de subvention $n^{o} 834759$ ).

\footnotetext{
${ }^{12}$ Laugier, S. Raison Publique n¹1, 2009, "Les séries télévisées : éthique du care et adresse au public"

${ }^{13}$ Foucault et alii., Les machines à guérir, 1995, Mardaga

${ }^{14}$ Hippocrate, Des épidémies, I, 5, 2016, Les belles lettres
} 